\title{
Análisis comparativo de fuentes bibliográficas web respecto la técnica de energy harvesting/scavenging aplicada a nodos inalámbricos
}

\author{
Fernando Alvarez Blanco¹, Mauricio Vladimir Peña Giraldo²
}

Recibido: Agosto 19 de 2014 Aprobado: Diciembre 12 de 2014

\section{Resumen:}

El propósito de este artículo es presentar los resultados de la revisión bibliográfica desarrollada respecto al tema de las técnicas de energy havervesting o energy scavenging, teniendo como interés principal las aplicaciones y desarrollos en donde se implementan energías de tipo termoeléctrico y fotovoltaico en nodos de sensores inalámbricos. Los resultados de la revisión muestran un auge en el interés por este tipo de técnicas, encontrando gran diversidad de fuentes de energía, métodos de implementación y optimización, así como también un desarrollo avanzado de algoritmos de enrutamiento y de supervisión de energía en lo referente a nodos inalámbricos.

Palabras clave: Energy harvesting, Energy scavenging, Nodo inalámbrico, ZigBee, Generador termoeléctrico.

\section{Abstract:}

The aim of this article is to show the results of the bibliographic review developed in the field of energy harvesting and energy scavenging techniques, having as a main focus those applications and researches in which the thermoelectric and photovoltaic energy sources in wireless nodes are applied. The results of this revision shows a growing interest in those techniques, finding a great diversity of energy sources, methods for deployment and optimization, as well as an advanced development of routing algorithms and energy monitoring regard the wireless nodes.

Keywords: Energy harvesting, Energy scavenging, Wireless node, ZigBee, Thermoelectric generator.

\footnotetext{
${ }^{1}$ Ingeniero Electrónico, especialista en Telecomunicaciones Móviles, docente del Programa de Tecnología en Electrónica UNIMINUTO Sede Principal, pertenece al grupo de Investigación IT.

2 Ingeniero Mecánico, magister en Automatización Industrial, docente investigador de la Universidad Libre de Colombia sede Bogotá.
} 


\section{INTRODUCCIÓN}

Con la aplicación de la técnica conocida como energy harvesting/scavenging se busca que diversos transductores capten energía del ambiente en el sitio en donde se implementará algún dispositivo o equipo electrónico, de modo que estos puedan tener un tiempo de funcionamiento prolongado con autonomías adecuadas y para diversas aplicaciones (Costis Kompis, 2008; Murugavel, 2008; Roy Yarza \& Catalán López, 2011).

Mediante esta revisión bibliográfica se presenta un referente en el desarrollo de la técnica energy havervesting/scavenging aplicada a nodos inalámbricos, con un enfoque particular en los nodos tipo ZigBee, de forma que permita contar con una aproximación global al estado del arte, reduciendo a su vez el tiempo de la consulta previa al desarrollo de proyectos en el área. Esta revisión está fundamentada en otros artículos en donde se han desarrollado recopilaciones del estado del arte y revisiones bibliográficas (Costis Kompis, 2008; Harb, 2011), lo que a su vez es un indicativo del interés en el desarrollo de esta técnica.

\section{METODOLOGÍA}

La presente revisión bibliográfica cubre el periodo comprendido entre los años 2007 y 2013. Se utilizaron las bases de datos en línea ScienceDirect (http:// www.sciencedirect.com/), Springer Link(http:// link. springer.com/) y la base Xplore de la IEEE (http:// ieeexplore.ieee.org/Xplore/guesthome.jsp) referenciando los siguientes términos de búsqueda: energy harvesting, energy scavenging, wireless sensor node, ZigBee, thermoelectric generator y TEG. La aclaración de algunos términos y conceptos relacionados con la técnica se realizó en el buscador Google y mediante consulta en literatura especializada en cada tema en particular.

Para restringir los resultados de la temática de interés, en todas las bases de datos se hizo la búsqueda directa de la expresión "energy harvesting scavenging" desde el año 2007 al 2013, filtrando los resultados en las bases de datos Springerlink y Science direct con los siguientes términos:

- Springerlink: energy harvesting scavenging, filtrado por: disciplina: ingeniería, subdisciplina: electrónica.

- Science direct: energy harvesting, energy harvester, wireless sensor, sensor network, electron transport, uv-b radiation.

En la base de datos Xplore de la IEEE no se filtraron los resultados dado que esta es una base especializada en electrónica.

\section{RESULTADOS OBTENIDOS}

Como resultado inicial de la búsqueda bibliográfica, en la Tabla No. 1 se presenta un resumen de los artículos encontrados con respecto a la cantidad de publicaciones por año. Se observa un interés creciente en el desarrollo de la técnica energy harvesting / scavenging en el periodo de tiempo definido para la revisión bibliográfica, pero que no es tan importante en los años 2007 y 2008.

Tabla 1: Cantidad de artículos / año en las bases de datos consultadas

\begin{tabular}{|c|c|c|c|c|c|c|c|c|}
\hline \multirow[t]{2}{*}{ BASE DE DATOS } & \multicolumn{7}{|c|}{ AÑO } & \multirow[t]{2}{*}{ TOTAL } \\
\hline & 2007 & 2008 & 2009 & 2010 & 2011 & 2012 & 2013 & \\
\hline SPRINGERLINK & 8 & 9 & 9 & 10 & 13 & 10 & 7 & 66 \\
\hline Science Direct & 1 & 4 & 4 & 10 & 22 & 24 & 19 & 84 \\
\hline Xplore IEEE & 17 & 33 & 42 & 39 & 55 & 50 & 46 & 282 \\
\hline
\end{tabular}

Las búsquedas anteriores arrojaron resultados muy diversos en cuanto el tipo de energía utilizado, el manejo de la fuente de energía o del protocolo de comunicación, el desarrollo de materiales para captación de energía y aplicaciones de la técnica energy havervesting/scavenging en diversos ámbitos, entre otros. Por lo anterior solo se con- templaron los resultados que tuvieron relación con fuentes de energía fotovoltaica, termoeléctrica, mecánica y radio - frecuencia. A continuación se hace un breve análisis de los resultados desde la perspectiva del tipo de energía captado, el algoritmo de optimización y las aplicaciones en nodos inalámbricos. 


\subsection{Comparativo de fuentes de energía}

Uno de los aspectos analizados en esta revisión bibliográfica es la clasificación de las búsquedas según el tipo de energía, dado que esto revela el interés por el desarrollo de alguna de las alternativas mencionadas previamente en este artículo. En la Tabla No. 2 se relacionan los hallazgos en las bases de datos consultadas con respecto al tipo de energía captada.

\begin{tabular}{|l|c|c|c|c|}
\hline \multirow{2}{*}{\multicolumn{1}{c|}{ BASE DE DATOS }} & \multicolumn{4}{c|}{ Tabla 2: Cantidad de referencias por tipo de energía } \\
\cline { 2 - 6 } & Fotovoltaica & Termoeléctrica & Electro - Mecánica & RF \\
\hline SPRINGERLINK & 1 & 1 & 20 & 0 \\
\hline Science Direct & 1 & 0 & 33 & 4 \\
\hline Xplore IEEE & 13 & 16 & 75 & 28 \\
\hline Fuente: los autores & \multicolumn{4}{c}{} \\
\hline
\end{tabular}

La tabla anterior incluye también resultados de proyectos en donde se hicieron desarrollos o experimentaciones con más de una fuente de energía a la vez. La cantidad de artículos por cada base de datos difiere con respecto a los de la Tabla No. 1 debido a que la búsqueda global incluyó términos como redes de sensores inalámbricos, protocolos de funcionamiento y revisiones del estado del arte, entre otros.

El uso de diversos medios de almacenamiento de la energía captada es otro de los temas de interés en los artículos consultados. Estos medios de almacenamiento van desde baterías recargables, generalmente de Li-Ion (Belleville et al., 2010) o de NiMH (Alippi \& Galperti, 2008), baterías de película plana (Anton, Erturk, Na Kong, \& Inman, 2009; Dudney, 2009; Roy Yarza \& Catalán López, 2011; Wang, Wang, Hayes, O’Flynn, \& Ó Mathúna, 2011), supercapacitores (Alex S. Weddell, 2008; Brunelli, Benini, Moser, \& Thiele, 2008; «EDN - Coupling a supercapacitor with a small energy-harvesting source| EDN», s. f.; Weddell, Merrett, \& Al-Hashimi, 2012) e incluso se encuentran referencias de trabajos con utilización de métodos de almacenamiento híbridos en donde se usan simultáneamente supercapacitores y baterías (ya sea de Li-lon o baterías de película plana) (Jessen, Venzke, \& Turau, s. f.; Wang et al., 2011), para almacenar una mayor cantidad de energía y aumentar la disponibilidad de ésta para el dispositivo conectado al sistema de captura almacenamiento de energía, así conformado.

\subsection{Algoritmos de optimización}

Un elemento común en muchos artículos consultados respecto a la técnica de energy havervesting/ scavenging, es la experimentación con diversos métodos para capturar la mayor cantidad de energía posible con un tipo de transductor dado. Esto es, buscar la forma de aprovechar al máximo la eficiencia propia del captador de energía (como por ejemplo, una celda fotovoltaica o un transductor piezoeléctrico) para lo cual se observa un creciente interés en la implementación de métodos de seguimiento del punto de máxima potencia a diversos dispositivos y formas de capturar la energía (MPPT por sus siglas en ingles - Maximum Power Point Tracking) (Alippi \& Galperti, 2008; Brunelli et al., 2008; Jessen et al., s. f.; Laird, Lovatt, Savvides, Lu, \& Agelidis, 2008; Shi, Miller, Mayaram, \& Fiez, 2011; Tan \& Panda, 2011; Weddell et al., 2012). En la Tabla No. 3 se muestra un comparativo de algunos tipos de algoritmos MPPT aplicados a celdas fotovoltaicas.

Tabla 3: Comparación de algoritmos MPPT para celdas fotovoltaicas (Onat, 2010)

\begin{tabular}{|l|c|c|c|c|c|c|}
\hline \multirow{2}{*}{\multicolumn{1}{|c|}{ COMPARISON PARAMETERS }} & \multicolumn{5}{c|}{ MPPT Algorithms } \\
\cline { 2 - 7 } & $\begin{array}{c}\text { Perturb \& } \\
\text { onserve }\end{array}$ & $\begin{array}{c}\text { Modified } \\
\text { P\&O }\end{array}$ & $\begin{array}{c}\text { Artificial } \\
\text { intelligence }\end{array}$ & $\begin{array}{c}\text { Constant } \\
\text { voltage } \\
\text { (current) }\end{array}$ & $\begin{array}{c}\text { Incremental } \\
\text { conductance }\end{array}$ & $\begin{array}{c}\text { Parasitic } \\
\text { capacity }\end{array}$ \\
\hline Efficiency (\%) & $81.5-85$ & $93-96$ & $>95$ & $88-89.9$ & $73-85$ & 99.8 \\
\hline PV Panel depending operation & No & No & Yes & Yes & No & No \\
\hline Exactly MPP determination & Yes & Yes & Yes & No & Yes & Yes \\
\hline Analog or digital control & Both & Digital & Both & Analog & Digital & Analog \\
\hline
\end{tabular}




\begin{tabular}{|l|c|c|c|c|c|c|}
\hline \multirow{2}{*}{ COMPARISON PARAMETERS } & \multicolumn{5}{|c|}{ MPPT Algorithms } \\
\cline { 2 - 7 } & $\begin{array}{c}\text { Perturb \& } \\
\text { onserve }\end{array}$ & $\begin{array}{c}\text { Modified } \\
\text { P\&O }\end{array}$ & $\begin{array}{c}\text { Artificial } \\
\text { intelligence }\end{array}$ & $\begin{array}{c}\text { Constant } \\
\text { voltage } \\
\text { (current) }\end{array}$ & $\begin{array}{c}\text { Incremental } \\
\text { conductance }\end{array}$ & $\begin{array}{c}\text { Parasitic } \\
\text { capacity }\end{array}$ \\
\hline Periodic tuning requierement & No & No & No & Yes & No & No \\
\hline Convergence speed & Varies & Fast & Fast & Medium & Varies & Fast \\
\hline Complexity & Low & Medium & High & Low & Medium & Low \\
\hline Measured parameters & $\begin{array}{c}\text { Voltage, } \\
\text { current }\end{array}$ & $\begin{array}{c}\text { Voltage, } \\
\text { current }\end{array}$ & Varies & $\begin{array}{c}\text { Voltage } \\
\text { (current) }\end{array}$ & $\begin{array}{c}\text { Voltage, } \\
\text { current }\end{array}$ & Voltage, \\
current
\end{tabular}

Es de notar que, a pesar de que la implementación de la técnica de MPPT es aplicable a cualquier elemento captador de energía, se ha encontrado que el desarrollo experimental de muchos artículos aplican esta técnica a captadores fotovoltáicos (A. Yafaoui., R. Cheung, \& B. Wu, 2007; A.J. Mahdi, W.H. Tang, \& Q.H. Wu, 2010; J. Ahmad \& Kim, s. f., s. f.; Ali, Saied, Mostafa, \& Abdel-Moneim, 2012; 2008; Masahito Shoyama, 2011; Esram \& Chapman, 2007; Onat, 2010, 2010; Sankman \& Ma, 2011; Sera \& Sera, 2009, 2009; Vieira \& Mota, 2010), y ello con respecto a otra técnicas como captadores termoeléctricos (Laird et al., 2008; Zhou, 2007), y fuentes electro-mecánicas (Kong \& Ha, 2012; Sankman \& Ma, 2011; Yi, Su, Lam, Ki, \& Tsui, 2008), entre otras.

Con relación a la cantidad de artículos que mencionan los diferentes tipos de captadores de energía con respecto a la técnica de MPPT, se realizó la búsqueda de este término combinado con las palabras "photovoltaic", "thermoeléctic" y "piezoelectric" a fin de realizar la comparación en la cantidad de resultados arrojados por una base de datos especializada, en el rango de fechas establecido para esta revisión bibliográfica. Los resultados de esta comparación para la librería digital IEEE Xplore fueron: 1210 resultados para la combinación "MPPT""photovoltaic", 27 resultados para la combinación
"MPРT" - "thermoelectic" y 12 resultados para la combinación "MPPT" - "piezoelectric".

\subsection{Nodos sensores inalámbricos}

En los párrafos anteriores se hizo mención de aquellos artículos cuya temática se centra en capturar y almacenar la mayor cantidad de energía para el dispositivo que dependa del sistema de energy havervesting/scavenging, como proveedor de energía para su funcionamiento. Con respecto al tema de los nodos inalámbricos se encuentran trabajos en donde el enfoque principal es el protocolo de funcionamiento, buscando que el nodo inalámbrico opere solo en intervalos de tiempo limitados para optimizar de esta forma la cantidad de energía consumida por dichos dispositivos (Eu, Tan, \& Seah, 2010; Koulali, Kobbane, Koutbi, Tembine, \& Ben-Othman, 2012; C. Moser, Brunelli, Thiele, \& Benini, s. f.; Clemens Moser, Chen, \& Thiele, 2009; Tran-Thanh, Rogers, \& Jennings, 2012).

Uno de los principales aspectos a analizar de los dispositivos electrónicos denominados nodos sensores inalámbricos, son sus características de consumo y eficiencia energética, para lo cual Phil Smith ("Comparing Low-Power Wireless Technologies - Wireless Solutions | Digikey Technology Zones», s. f.) presenta las siguientes cifras características de algunos de estos dispositivos.

\begin{tabular}{|l|c|c|c|c|c|c|}
\hline \multicolumn{7}{|c|}{ Tabla 4: Comparación de diferentes tecnologías inalámbricas } \\
\hline & ANT & $\begin{array}{c}\text { Bluetooth de } \\
\text { baja energía }\end{array}$ & IrDA & Nike+ & Wi-Fi & ZigBee \\
\hline Throughput $^{3}$ & $\sim 20 \mathrm{kbps}$ & $\sim 305 \mathrm{kbps}$ & $\sim 1 \mathrm{Gbps}$ & $\sim 272 \mathrm{bps}$ & $\sim 6 \mathrm{Mbps}$ & $\sim 100 \mathrm{kbps}$ \\
\hline Consumo $(\mathrm{mW})$ & 0,183 & 0,147 & 0,163 & 0,675 & 210 & 35,7 \\
\hline Potencia por bit $(\mu \mathrm{W} / \mathrm{bit})$ & 0,71 & 0,153 & 11,7 & 2,48 & 0,005 & 185,9 \\
\hline Fuente: («Comparing Low-Power Wireless Technologies - Wireless Solutions | Digikey Technology Zones», s. f.) \\
\hline
\end{tabular}

\footnotetext{
${ }^{3}$ Se llama "throughput" a la tasa promedio de éxito en la entrega de un mensaje sobre un canal de comunicación. Tomado de: Conceptos básicos sobre planificación de redes, en: Diario de Planificación y Diseño de Redes y Servicios. https://diarioredesyservicios.wordpress. com/2012/01/11/conceptos-basicos-sobre-planificacion-de-redes/
} 
La tabla anterior es un resumen de las tecnologías inalámbricas comerciales con respecto a su consumo y tasa de transmisión de datos, pero no contempla el grado de interés para la implementación en conjunto con la técnica energy harvesting / scavenging. Para analizar este interés se recurrió nuevamente a la librería digital IEEE Xplore combinando los términos "energy havervesting scavenging" con cada una de las tecnologías enlistadas en la tabla anterior. La búsqueda fue realizada por la opción "Advanced search" filtrada con los términos: "harvesting" OR "scavenging" AND "energy" AND (la tecnología ANT, Bluettoth, WiFi, IrDA, NIKE+, o Zigbee en cada caso).

Con respecto a los resultados de esta búsqueda se obtuvieron datos poco confiables para los términos ANT, WIFI y Bluetooth, ya que para el primero la base de datos consultada arroja resultados relacionados con el algoritmo "ant" (hormiga) de enrutamiento de información y no con la tecnología ANT, propiedad de la empresa Dynastream que es el caso consultado; para los términos WiFi y Bluetooth se encontraron 11 resultados para cada uno, en donde se usan las ondas electromagnéticas generadas como fuente de energía, pero sin mencionar la forma de implementar un algoritmo de optimización en el manejo de la energía demandada por éste. Para las tecnologías IrDA y Nike+ no se obtuvieron resultados, mientras que para la tecnología ZigBee se presentaron 26 resultados, de los cuales alrededor de 12 de estos artículos tienen relación directa con la eficiencia en los protocolos de comunicación o con el uso combinado de estos dispositivos con sistemas de captura - almacenamiento de energía, que hacen uso de la técnica energy harvesting/scavenging.

\section{CONCLUSIONES}

En ésta revisión bibliográfica se ha observado un creciente interés por optimizar la captura y por el aprovechamiento de la energía proveniente de un gran número de fuentes, incluyendo entre otras vibraciones mecánicas (Anton et al., 2009; Gilbert \& Balouchi, 2008; Lallart et al., 2008), diferencias de temperatura (Cuadras, Gasulla, \& Ferrari, 2010; Kadirvel et al., 2012; Knight \& Davidson, 2010; Laird et al., 2008; Mousoulis, Yoon, Chitnis, \& Ziaie, 2012; Shi et al., 2011; "Turning Waste Heat into Electricity on the Nanoscale», s. f.; Zhou, 2007), campos electromagnéticos generados por equipos de comunicación (M. A. Ahmad \& Alshareef, 2012; Aminurrahim bin Othman, May; Huang, Doraiswami, Osterman, \& Pecht, June), y energía fotovoltaica (Alippi \& Galperti, 2008; Brunelli et al., 2008; Kadirvel et al., 2012; Lu \& Yang, 2009; Onat, 2010;
«Power from Two Energy Sources?», s. f.-a; Shi et al., 2011; Tan \& Panda, 2011).

En la revisión de la bibliografía se encuentran dos enfoques principales en el tema de manejo de energía en nodos sensores inalámbricos: uno de estos consiste en mejorar, tanto la captación de energía con diferentes combinaciones de algoritmos y circuitos como la disponibilidad de esta, almacenándola en distintos tipos de baterías y super-capacitores; el otro enfoque consiste en optimizar los regímenes de comunicaciones del nodo para demandar una menor cantidad de energía del sistema de captura almacenamiento.

Otra de las conclusiones surge al analizar la Tabla No. 2 en donde se observa un marcado interés por las fuentes de energía mecánica, las cuales pueden incluir elementos piezoeléctricos, MEMs (Micro Electro Mecánicos) y sistemas inductivos de generación de energía por medio del movimiento. También es de observar que, en contra de lo esperado, las fuentes de energía fotovoltaicas presentan en general un bajo interés en su desarrollo, quizás debido a que son del tipo de captador de energía que más tiempo de desarrollo ha tenido, comparativamente con las fuentes termoeléctricas, electromecánicas y de radio-frecuencia. Sin embargo, el conocimiento amplio acerca de las celdas fotovoltaicas las hace muy útiles para nuevos planteamientos de la técnica energy harvesting/scavenging, en donde el enfoque principal no dependa de hacer desarrollos complejos relacionados con el captador de energía.

En cuanto a las bases de datos consultadas, se observa que para el tema tratado la base de datos IEEE Xplore constituye la mejor alternativa respecto a bases de datos convencionales como SpringerLink y Science Direct, dada la cantidad y variedad de resultados que presenta en el tema de energy harvesting/scavenging. Esto fue muy útil al momento de analizar los distintos protocolos inalámbricos que son utilizados actualmente por la industria, puesto que únicamente con los datos de consumo y tasa de transmisión de información, no es suficiente para concluir cuál de estos protocolos es el más apropiado para ser incluido en un proyecto que combine la técnica de energy harvesting/scavenging, con nodos inalámbricos.

\section{REFERENCIAS BIBLIOGRÁFICAS}

1. A. Yafaoui., R. Cheung, \& B. Wu. (2007). IMPLEMENTATION OF MAXIMUM POWER POINT TRACKING ALGORITHM FOR RESIDENTIAL 
PHOTOVOLTAIC SYSTEMS. 2nd Canadian Solar Buildings Conference, 6.

2. A.J. Mahdi, W.H. Tang, \& Q.H. Wu. (2010). Improvement of a MPPT Algorithm for PV Systems and Its Experimental Validation (p. 6). Presentado en International Conference on Renewable Energies and Power Quality (ICREPQ'10), Granada (Spain). Recuperado a partir de http: // www.icrepq.com/icrepq\%2710/419-Mahdi.pdf

3. Ahmad, J., \& Kim, H. (s. f.). A Voltage Based Maximum Power Point Tracker for Low Power and Low Cost Photovoltaic Applications.

4. Ahmad, M. A., \& Alshareef, H. N. (2012). Energy harvesting from radio frequency propagation using piezoelectric cantilevers. Solid-State Electronics, 68, 13-17. doi:10.1016/j.sse.2011. 09.007

5. Alex S. Weddell, G. V. M. (2008). Energy Harvesting and Management for Wireless Autonomous Sensors. e-prints Soton Universidad de Southampton. Recuperado a partir de http: / / eprints.soton.ac.uk/265342/

6. Ali, A. N. A., Saied, M. H., Mostafa, M. Z., \& Abdel-Moneim, T. M. (2012). A survey of maximum PPT techniques of PV systems. En 2012 IEEE Energytech (pp. 1-17). doi:10.1109/ EnergyTech.2012.6304652

7. Alippi, C., \& Galperti, C. (2008). An Adaptive System for Optimal Solar Energy Harvesting in Wireless Sensor Network Nodes. IEEE Transactions on Circuits and Systems I: Regular Papers, 55(6), 1742 - 1750. doi:10.1109/TCSI.2008.922023

8. Amine DAOUD, \& Abdelhamid MIDOUN. (2008). Single Sensor Based Photovoltaic Maximum Power Point Tracking Technique for Solar Water Pumping System. Electrical Power Quality and Utilisation, Journal, Vol. XIV(No. 2), 69-72.

9. Aminurrahim bin Othman, M. (May). Waste of radio frequency signal analysis for wireless energy harvester. En 2010 6th International Colloquium on Signal Processing and Its Applications (CSPA) (pp. 1-3). doi:10.1109/CSPA.2010.5545241

10. Anton, S. R., Erturk, A., Na Kong, D. S. H., \& Inman, D. J. (2009). SELF-CHARGING STRUCTURES USING PIEZOCERAMICS AND THINFILM BATTERIES. Presentado en Proceedings of the ASME 2009 Conference on Smart Materials, Adaptive Structures and Intelligent Systems, Oxnard, California, USA. Recuperado a partir de http://www.vtvt.ece.vt.edu/research/papers/ 09SMASIS.pdf

11. Belleville, M., Fanet, H., Fiorini, P., Nicole, P., Pelgrom, M. J. M., Piguet, C., ... Cantatore, E. (2010). Energy autonomous sensor systems: Towards a ubiquitous sensor technology. Microelectronics Journal, 41(11), 740-745. doi:10.1016/j.mejo.2010.01.009

12. Brunelli, D., Benini, L., Moser, C., \& Thiele, L. (2008). An Efficient Solar Energy Harvester for Wireless Sensor Nodes. En Design, Automation and Test in Europe, 2008. DATE '08 (pp. 104 -109). doi:10.1109/DATE.2008.4484670

13. Comparing Low-Power Wireless Technologies Wireless Solutions | Digikey Technology Zones. (s. f.). Recuperado 29 de noviembre de 2012, a partir de http://www.digikey.com/us/en/tech zone/wireless/resources/articles/comparinglow-power-wireless.html

14. Costis Kompis, S. A. (2008). Energy Harvesting Technologies to enable Remote and wireless Sensing. Vodera Ltd and Zartech Ltd.

15. Cuadras, A., Gasulla, M., \& Ferrari, V. (2010). Thermal energy harvesting through pyroelectricity. Sensors and Actuators A: Physical, 158 (1), 132-139. doi:10.1016/j.sna.2009.12.018

16. Dudney, N. J. (2009). Thin Film Batteries for Energy Harvesting. En S. Priya \& D. J. Inman (Eds.), Energy Harvesting Technologies (pp. 355-363). Springer US. Recuperado a partir de http: / /www.springerlink.com/content/t7777 945488m70h8/abstract/

17. EDN - Coupling a supercapacitor with a small energy-harvesting source | EDN. (s. f.). EDN. Recuperado 10 de julio de 2012, a partir de http://www.edn.com/design/components-andpackaging/4374932/Coupling-a-supercapacitorwith-a-small-energy-harvesting-source-

18. Emad M. Ahmed, \& Masahito Shoyama. (2011). Variable Step Size Maximum Power Point Tracker Using a Single Variable for Stand-alone Battery Storage PV Systems. Journal of Power Electronics, Vol. 11(No. 2), 218-227.

19. Esram, T., \& Chapman, P. L. (2007). Comparison of Photovoltaic Array Maximum Power Point Tracking Techniques. IEEE Transactions on Energy Conversion, 22(2), 439-449. doi:10.1109/TEC. 2006.874230

20. Eu, Z. A., Tan, H.-P., \& Seah, W. K. G. (2010). Opportunistic routing in wireless sensor networks powered by ambient energy harvesting. Computer 
Networks, 54(17), 2943-2966. doi:10.1016/j.com net.2010.05.012

21. Gilbert, J., \& Balouchi, F. (2008). Comparison of energy harvesting systems for wireless sensor networks. International Journal of Automation and Computing, 5(4), 334-347. doi:10.1007/s11 633-008-0334-2

22. Harb, A. (2011). Energy harvesting: State-ofthe-art. Renewable Energy, 36(10), 2641-2654. doi:10.1016/j.renene.2010.06.014
23. Huang, Y., Doraiswami, R., Osterman, M., \& Pecht, M. (June). Energy harvesting using RF MEMS. En Electronic Components and Technology Conference (ECTC), 2010 Proceedings 60th (pp. 1353-1358). doi:10.1109/ECTC.2010.5490638

24. Jessen, J., Venzke, M., \& Turau, V. (s. f.). Design Considerations for a Universal Smart Energy Module for Energy Harvesting in Wireless Sensor Networks. Recuperado a partir de http://130. 203.133.150/viewdoc/ 